\title{
Asset Management System Processes: Implementation of Sensor and Artificial Intelligence
}

\section{Donald Levi Tryon*}

The Graduate College at the University of Nebraska in Partial Fulfillment of Requirements, Lincoln, Nebraska, USA

\begin{abstract}
This is a review of asset management systems and their implementations of sensor and artificial intelligence. There are many ways to implement management systems to help track the life cycle of assets within an organization. These systems can be upgraded with the use of sensors to collect live parameters of the asset's current conditions to analyze their current state of operation. From here, the conditional state of operations should engage an action from the organization's business model to cause an event. There are examples offered in this paper from alternative research that depicts the benefits of implementation of both sensors and artificial intelligence algorithms within the organizations. This is an overall review of how these interactions can be processed and implemented. Future studies will need to be conducted in the areas of methods and processes, in particular, in order to integrate a generic model for sensors and artificial intelligence into successful asset management systems.
\end{abstract}

Keywords: Asset management system; Artificial intelligence

\section{Overview}

The world is changing in the way it gathers sensory information. The world is becoming more digitally interfaced; this digitally interfaced world is creating the requirement for reevaluating asset systems. In the past, asset systems have been a place to take inventory of objects that have been used or that are currently being used by an organization. The new digital systems use software tracking to help manage all of the assets. However, rather than simply tracking the current state of any asset; would it be possible to use a digitally improved form of asset management systems to prevent catastrophic failures?

There are ways to prevent failures when modern sensors are implemented correctly. This paper will provide a breakdown of how current systems are using asset management systems, the benefits of such, and examples and reasoning for implementation. The paper begins with a literature review of asset management systems; this is to ensure the systems are functional and correctly implemented. The methodology section will review ways to use sensors and artificial intelligence to predict failures. The last section will provide the conclusion of implementation results and outcomes.

\section{Literature Review}

There is currently a vast amount of existing literature on the topic of asset management systems. Asset management is originally decades old, but now is taking on a new, profound meaning as it grows from a minimal side-thought to a strong contributor of any business strategy model [1]. Each of the literature provides beneficial information to develop a strong fundamental understanding of: 1) defining asset management systems, 2) the reason for asset management systems, 3) ways to implement management systems, and 4) examples of asset systems that have been implemented correctly. This report will focus on the construction of asset management systems while highlighting the four listed fundamentals for asset management.

\section{Defining asset management and asset management systems}

The ISO 55000 (the International Organization for Standardization) defines asset management as "coordinated activity of an organization to realize value from assets" and defines assets as "an item, thing or entity that has potential or actual value to an organization [2]." Asset management encompasses a wide-range of areas from managing physical assets, to even financial, technical, human, information and intellectual assets. It is used to improve such things as, life cycle stage, maintenance planning, and the whole product-life view from creation to disposal of assets [1].

Another common definition to define asset management is presented by the British Standards Institution Publicly Available Specification [3] as "systematic and coordinated activities and practices through which an organization optimally and sustainably manages its assets and asset systems, their associated performance, risk and expenditures over their life cycles for the purpose of achieving its organizational strategic plan (British Standards Institution, 2008).'

Asset management systems are those systems that can use resources and direct them to make decisions on their own, normally through equations. These management systems would query data within the asset types and use the queries to make decisions based off of the instant results that are discovered. These systems are used for reordering, purchasing, and movement of assets, etc. Decision making models are sometimes referred to as Computerized Maintenance Management System (CMMS), Supervisory Control and Data Acquisition (SCADA), or IT based systems called IT Asset Management (ITAM). Asset management systems must use company designed rules and guidelines to make decisions to benefit the stakeholders and create smoother interaction within the business management definition.

\section{The reason for asset management systems}

Asset management systems allow for organizations to implement changes to help manage the assets that will increase value throughout

*Corresponding author: Donald Levi Tryon, The Graduate College at the University of Nebraska in Partial Fulfillment of Requirements, Lincoln, Nebraska, USA, Tel: (+1) 402871 6175; Fax: (+1) 402871 6175; E-mail: dlevitryon@gmail.com

Received September 07, 2017; Accepted October 06, 2017; Published October 11,2017

Citation: Tryon DL (2017) Asset Management System Processes: Implementation of Sensor and Artificial Intelligence. Ind Eng Manage 6: 231. doi:10.4172/21690316.1000231

Copyright: (c) 2017 Tryon DL. This is an open-access article distributed under the terms of the Creative Commons Attribution License, which permits unrestricted use, distribution, and reproduction in any medium, provided the original author and source are credited. 
Citation: Tryon DL (2017) Asset Management System Processes: Implementation of Sensor and Artificial Intelligence. Ind Eng Manage 6: 231. doi:10.4172/2169-0316.1000231

the entire business model directives. Some of the benefits that can come from implementing an asset management system are [4]:

- Improved financial performance: Improving the return on investments and reducing cost can be achieved, while preserving asset value and without sacrificing the short or longterm realization of organizations objectives;

- Informed asset investment decisions: Enabling the organization to improve its decision-making and effectively balance cost, risk, opportunities and performance;

- Managed risk: Reducing financial losses, improving health and safety, good will and reputation, minimizing environmental and social impact, can result in reduced liabilities such as insurance premiums, fines and penalties;

- Improved services and outputs: Assuring the performance of assets can lead to improved services or products that consistently meet or exceed the expectations of customers and stakeholders;

- Demonstrated social responsibility: Improving the organization's ability to, for example, reduce emissions, conserve resources and adapt to climate change, enables it to demonstrate socially responsible and ethical business practices and stewardship;

- Demonstrated compliance: Transparently conforming with legal, statutory and regulatory requirements, as well as adhering to asset management standards, policies and processes, can enable demonstration of compliance;

- Enhanced reputation: Through improved customer satisfaction, stakeholder awareness and confidence;

- Improved organizational sustainability: Effectively managing short and long-term effects, expenditures and performance, can improve the sustainability of operations and the organization;

- Improved efficiency and effectiveness: Reviewing and improving processes, procedures and asset performance can improve efficiency and effectiveness, and the achievement of organizational objectives.

The British Standards Institution Publicly Available Specification also suggests that some of the areas to benefit from a successful implementation are: improved corporate reputation which may enhance shareholder value, optimized return on investment and/or growth, and enhanced customer satisfaction from improved performance and control from product or service delivery to the required standards [5].

There are numerous reasons for corporations to be interested in improving the business plan by implementing a successful asset management plan. It is important to recognize the need for leadership involvement when implementing directives in order to be successful when using asset management systems. Without the appropriate and clear leadership directives, benefits gained will be minimal.

\section{Ways to implement management systems}

Asset management systems can cover a wide-range of resources, anything from hardware to employees, which may change how the systems will be laid out, but ultimately does not change the fundamentals needed to create a successful system.

British standards institution publicly available specification: British Standards Institution Publicly Available Specification originally suggested the key principles and attributes in the design and implementation of the asset management systems should be: 1) holistic, 2) systematic, 3) systemic, 4) risk-based, 5) optimal, 6) sustainable, and 7) integrated [5]. In the figure below you can see the relationship of the principles (Figure 1).

British Standards Institution Publicly Available Specification goes on to explain each of the seven key principles to implement a successful asset management system.

Holistic: Holistic is looking at the larger picture and asking; can any of the parts be combined or joined with another asset? One way holistic can be implemented is to integrate other systems with data from the asset management systems to dictate other tasks. An example of a holistic approach is to design a form of work management system that works within the asset system; some of these systems are called Computerized Maintenance Management Systems (CMMS). Chau et al. explains that "the Work Management of CMMS is purposed to ensure that maintenance personnel can manage and plan incoming work request as well as automatically generated work from preventive maintenance programs [6]."

Having the holistic principle in mind, they used the main asset management system to drive other work processes from computer generated results to have a more uniform work flow.

Systematic: Systematic is producing an approach that is a repeatable action which is auditable; can this step be reproduced and measured for quality? One of the main ways to ensure quality is to equip the assets with a form of sensors which will measure any required auditable information needed. Some of the parameters that can be captured by current sensors are, "operational and environmental loads as well as the performance conditions of the product, for example, temperature, vibration, shock, pressure, acoustic levels, strain, stress, voltage, current, humidity levels, contaminant concentration, usage frequency, usage severity, usage time, power and heat dissipation [7].'

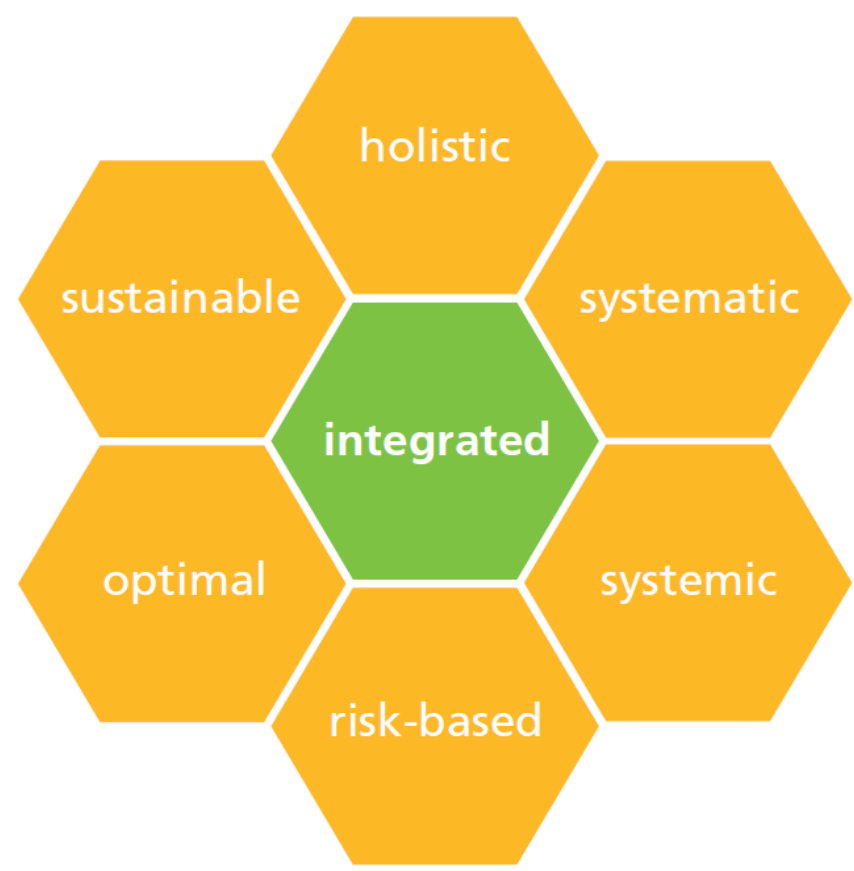

Figure 1: Key principles and attributes of asset management [2] 
One method that uses the parameters to measure quality implements measurements and sensors in order to use a form of prognostics and health management (PHM); PHM "generally combines sensing and interpretation of environmental, operational, and performance-related parameters to assess the health of a product and predict remaining useful life [7]." To enhance the systematic measures, the measurements should be taken in real-time so actions can be added to the current work flow using a form of a computerized maintenance system with the integrated Supervisory Control and Data Acquisition (SCADA).

SCADA is a system to "realize and implement various functions such as creating, linking, triggering and maintaining corrective maintenance and preventive maintenance task [6]." Using the systematic approach will allow quality of view with the current asset states, which will limit downtime failures.

Systemic: Systemic involves reviewing the asset so it can be optimized and reduce cost and risk by means of isolation; will this asset cause a reduction in service if it fails within the system? British Standards Institution Publicly Available Specification explains that it may not be a reasonable request to provide a risk evaluation for every asset and asset system owned by an organization, but more practically to focus on significant risks, with lesser degrees of reporting and control required for lower risk areas. To overcome this concern, it is suggested that it may be "necessary for the organization to be able to consider similar risk from across the system so that the overall impact on the business can be evaluated, and the most appropriate organization-wide controls can be put into place, e.g. standard work or common procedures [5]." Using standard documents to overcome system variability will help the systemic principle of the organizations.

Creating standards for the organization will "ensure value is generated for the stakeholders [8]." Standards can be created to outline maintenance on a particular machine or standards in data collection and displays. Mahmoud Halfawy expresses the benefits of standardized data collection, "some of these models have reached a high level of maturity that offered the industry an unprecedented ability to represent, integrate, and share data. The success and wide adoption of these models is strong evidence of the need for feasibility of developing comprehensive data standards in the construction industry [9]." Standards facilitate the systemic principle by allowing the data to be sent to other organizations within the supply chain to help limit the risk of certain failures that can occur, like outages or supply shortages.

Risk-based: Risk-based is explained as "focusing resources and expenditures, and setting priorities, appropriate to identified risk and the associated cost/benefits [5];" if there is a failure in the asset, are there processes in place to counteract the issues that may arise? Cheng et al. state that using the prognostic and health management (PHM) "during all stages of the product life cycle, including manufacturing, shipment, storage, handling, and operations, [is essential] since failures may occur due to the abnormal operational or environmental conditions of all these stages [7]." Chau et al. explains that it "is of vital importance that well-times maintenance and routine inspection of achieving an optimum service life of an asset with aims of expert knowledge before catastrophic failures occur [6]." From the reading, the key way to reduce risk is to know where the weak points are of any system and focus on making it as efficient and safe as the rest of the assets in the system.

Optimal: Optimal is to ensure the asset is the best value; are there any alternative assets that are a better choice? One way to create optimal solutions is by making decisions and forecasting in real time. This can be completed with an array computer based equation which creates a form of condition based monitoring. A "condition based monitoring engine integrates real time plant information and assesses equipment status accurately and timely. It relies on rule based condition monitoring engine for creating work orders automatically that minimize human and capital resources [6]."

Sustainable: Sustainable means to view the long-term consequences of the current activities; will our actions today cost the organization more in the long run? It is important for an organization to review the long-term cost and benefits that an asset management system can generate, even if the current decision is cutting cost now. "Decisions like cost cuts usually support a shorter view...decisions on the basis of capital cost may seem [like these are] driving down expenses in the short term, however in the long term these may be driving baseline costs high and lower customer satisfaction [8]." In order for sustainability, an organization must see continued value from the asset management systems in-order to keep the processes in place.

Integration: Integration is combining resources to achieve the optimal solution; what resources do we have to help manage the current assets? Mahmoud Halfawy states that "an integrated approach to infrastructure management can potentially eliminate many of the fragmentation inefficiencies by enabling the integration of data and software resources, coordination of decision-making processes, and the efficient sharing and management of asset lifecycle data [9]." The integration can be solved by a two-step process. The first step is creating a coordinated, direct link to each of the shareholders that will be part of the infrastructure management process. Once all of the shareholders are in line, the second step is to define the scheme for the data and management styles. During the second stage, distributed information and software resources are reviewed and sorted for determining which system would be best to handle that data. From there, the standardized information can be integrated across all of the shareholders in the asset management systems.

International standard ISO: Besides the British Standards Institution Publicly Available Specification, the International Standard ISO 55001:2014 breaks the implementation process down into several different steps. These steps are: 1) understanding the organization, 2) understanding the needs and expectations of the stakeholders, 3 ) determining the scope of the asset management system, 4) asset management system, 5) leadership, and 6) plan [4]. The first step that ISO 55001 emphasizes is to understand the organization and the needs and expectations of the stakeholders. "Asset management objects, included in the strategic asset management plan (SAMP), shall be aligned to, and consistent with, the organization objectives [2]." For the objective of the management system, the importance must be stressed when determining the stakeholders and their requirements and expectations as the stakeholders. This helps when declaring ownership of responsibility of certain tasks to make a successful and sustainable system.

Once the stakeholders are selected, they must then determine the scope of the asset management system. This means the organization must "determine the boundaries and applicability of the asset management system [4]." This will create a structured and a focused directive to follow which will define the asset portfolio; it is recommended that the scope is in documented form. Once the owners and boundaries are declared, ISO 55001:2014 suggests it shall then "establish, implement and continually improve an asset management system, including the processes needed and their interactions... [2]". It is important to point out that only after the limits, the scope and ownership are declared before any asset management implementations occur. 
Leadership must provide support to the asset management systems by ensuring that the system is following organizational policies and that resources are available to continue support of the new system. Leadership must also ensure the organizational goals are being maintained, such as financial gains and effectiveness. IOS 55001:2014 says leadership should promote "cross-function collaboration within the organization" and "continual improvement [2]." This leads in to the plan stage of the implementation process; the plan is referred to when accounting for risks and opportunities. It is used to help account for problems that can break down the system, but also allows flexibility when seeking out areas which can add benefits to the system.

\section{Methodology}

There has been much advancement during the recent years that will allow asset management systems to run more efficiently by adding technical alternatives that will allow a reduction in time to complete a task. Some examples of a reduction in time are: user wait time, report interpretation/generation time, and initiation of action. In order to create some of these reductions, I will be looking at ways to implement sensors or artificial intelligence.

\section{Implementation of a sensor network}

Sensors can measure and test devices. The main benefit of sensors is they have the capability of reporting live information back into the organization systems. This type of configuration can allow real time reporting as it provides access to live measurements and information. Cheng et al. states that when sensors are integrated, data models can enable identification of "the deviation or degradation of a product from an expected normal condition and the prediction of the future state of reliability [7]." Cheng et al. used a prognostic and health management system (PHM) "which generally combines sensing and interpretation of environmental, operational and performance-related parameters to assess the health of a product and predict remaining useful life." This method of prognostics takes a large amount of sensor data to validate the norm of the equipment.

Cheng et al. presents three main areas in which the prognostics and health management systems must rely on. The first of the three areas requires monitoring large product parameters to evaluate the health of any product. These areas include operational and environmental loads as well as performance conditions. The second area is the collection of parameters for all stages of the product life, including manufacturing, shipment, storage, handling, and operation. Cheng et al. explains the importance for this "since failures may occur due to the abnormal operational or environmental conditions of all these stages [7]." Parameters for conditions should be collected by sensors. Lastly, the third area requires that sensors should be reliable and should have relatively low cost, so that it does not adversely affect the benefits of monitoring the products.

Reduction in time: The implementation of sensors with prognostics and health management systems save time by helping determine parameters that may cause future failures. Failures within systems cause undue wait times for both customers and organizations. These wait times can be the result of waiting for equipment to be repaired, waiting on products for the next stage, or customers waiting for completed products. Using predictive parameters to determine possible failures will prevent delays since preventive maintenance can be completed before total failure.

Sensor implementation is useful when collecting information parameters about current conditions that exist in a product. Once the information is collected, the data can be used to implement actions that will prevent further delays in the system. It is important to remember sensor information should be collected during all life cycle stages and to revisit predictive analysis to ensure quality has not changed.

Asset management systems with sensors: The use of sensors is an effective tool when incorporating them correctly into any asset management system. Using the British Standard PAS 55-2, the sensors must abide by the seven key principles and attributes of asset management.

That is to say the sensors must practice a holistic relationship with the systems to dictate task or allow for input in controls when engaging an action in the system chain. All of the sensors should be systemic and systematic with regards to recording the same information over the life cycle of the product and optimizing the value of the sensor. The sensor should not weaken the sustainable performance, increase cost, or add potential risk to the product. The implementation of sensors should be considered when reviewing risk-based and optimal additive value to the system. The sensor parameters should help identify risk of possible failure to optimize the "best value compromise between competing factors, such as performance, cost and risk, associated with the assets over their life cycles [5]." Sustainability is a key factor with successful implementation of sensors in a management system because it is important to consider the long-term and short-term activities that the parameters should collect as well as the future data requirements of those parameters. Finally, the sensors should be integrated, not only into products or tasks, but into the system as a whole. These integrated sensors should become interdependencies that drive actions to success. The data should be used for reaction but also proactive. An example of a reaction would be automatic scheduling once a failure is detected. An example of a proactive action would be preventive maintenance scheduling.

When adding sensors to an asset management system, it is important to view how the parameters can be used to create an interdependent relationship for enabling actions. These actions should reflect the proactive results desired by the organizational business plan. With a successful implementation plan, the results should be seamless with minimal requirements needed for deriving the results. The goal is to benefit the system and not to create more non-additive value to the system.

\section{Implementation of artificial intelligence}

Nemati et al. purposed the benefits of including artificial intelligence. It suggests "artificial intelligence is to amplify the cognitive capabilities of the decision maker in converting tacit knowledge into explicit knowledge, integrating this explicit knowledge by analyzing it to detect new patterns and relations, and understanding the new knowledge by providing analogs and explanations [10]." The implementation of artificial intelligence (AI) will help create an explicit knowledge of how a decision maker is making the end decision, and then create an algorithm in which a computer based system can make the same, or better, quality decisions without the intervention of a "tacit knowledge" user. This will allow seamless interactions between assets and decisions like maintenance planning.

One example of artificial intelligence and seamless interactions was presented by Chau et al. to the Drainage Service Department of the Government of the Hong Kong Special Administrative Region. Chau et al. mounted three sets of vibration sensors on the pump shaft of an outfall screw pump, and then developed a degrading index which was 
Citation: Tryon DL (2017) Asset Management System Processes: Implementation of Sensor and Artificial Intelligence. Ind Eng Manage 6: 231. doi:10.4172/2169-0316.1000231

Page 5 of 5

used to find the value of the current condition of the pump. From there, the group fed the results into the computerized maintenance management system (CMMS) that drove various functions such as "creating, linking, triggering and maintaining corrective maintenance and preventive maintenance tasks [6]." This hands-off approach allowed for more of an effective way to do an instant testing of equipment.

Reduction in time: Using AI to reduce the amount of "tacit knowledge" and implement standard routines that can apply controls to tasks will decrease the amount of lag between reporting and actions. Most organizations have personnel that acquire data in the form of a report, and then make predictions to apply actions and drive a task. However, with the implementation of AI to analyze data per an organization's directive, and then to initiate a task with no user required will reduce the lag time between data acquired and task. Depending on the task, the wait time can be reduced for the end user since there will be an elimination of steps. Using AI to generate reports will reduce the requirement to visually show information to a user (tacit knowledge) who would have then needed to interpret the data and make a decision. Now this can be expedited by the capability of the AI generated algorithm to complete the same task instantly.

Asset management systems with artificial intelligence: When using artificial intelligence to determine the algorithms for decision making, it is important to review the stakeholders and business module of the organization. The British Standard PAS 55-1 presents other considerations that are essential for a successful asset management system that works well in hand with AI methods. These considerations include having "adequate information and knowledge of asset condition, performance, risk and cost, and the interrelationships between these [5]." Without a clear understanding of the total conditions and interrelationships, the artificial intelligence algorithms may have higher than excepted errors. Implementation of AI should be part of a continuous improvement process with updated and larger data collected. This will lead to the best possible outcome for AI implementation.

An example for why an organization would want to implement an asset management system is in the Provance article, IT Asset Management: It's All about Process, states that “...clients who have successfully executed ITAM [11] as a discipline have typically achieved $30 \%$ cost savings in the first year, and at least $5 \%$ cost savings in each subsequent five years. [11]" The increase cost savings is directly related to a successfully implemented plan of the IT asset management system which, in this case, focused on the centralized management of financial, contractual, and physical data about IT assets in an organization [11].

Overall there are large amounts of research available for implementing sensors and AI into asset management systems. There are also benefits that an organization will receive when implementation is successful. It is important to discover which areas can receive the highest gains for the integration of changes into the network.

\section{Conclusion}

In conclusion there is a growing amount of research to show new ways to implement technology to receive benefits within asset management systems. The way systems are implemented into organizations can greatly affect whether or not the asset system will be successful. The new technology that is currently available can greatly improve the results and reduce the required resources for collecting and analyzing the data. Some areas that need to be investigated further are the benefits of sensors and artificial intelligence algorithms for certain asset parameters. The additive sensors and artificial intelligence will allow instant interactions between asset management systems like active maintenance planning. The ideal system that will need to be researched further is a system that implements live sensor parameters to feed into an artificial intelligence algorithm that will initiate an action with no user required input.

The large data sets create new ways to implement change with asset management systems that will help increase organization responsiveness and quality. It is important for an organization to review the areas that can benefit from live parameters collected from sensors and implement them into the business model. These implementations can reduce risk and help with future issues that may arise.

\section{References}

1. Smith TW (2014) Understanding the History, Foundations and Benefits of Asset Management. The Steven L Newman Real Estate Institute, Spring.

2. International Organization for Standardization (2014) 55000 - International Organization for Standardization, p:19

3. British Standards Institution (2008) PAS 55-1 Asset Management Part 1 Specification for the optimized management of physical assets, p: 24

4. International Organization for Standardization (2014) 55001 - International Organization for Standardization, p:14.

5. British Standards Institution (2008) PAS 55-2 Asset Management Part 2 Guidelines for the application of PAS 55-1.

6. Chau HKM, Li RC, Lee TS, Cheung BS, Loy TS (2015) Implementation of Computerized Maintenance Management System (CMMS) in Upgraded Pillar Point Sewage Treatment Works.

7. Cheng S, Azarian MH, Pecht MG (2010) Sensor Systems for Prognostics and Health Management. Sensors 10. 10: 5774-5797.

8. Urmetzer F, Parlikad AK, Pearson C, Andy N (2014) Design Considerations for Engineering Asset Management Systems. Lecture Notes in Control and Information Sciences.

9. Mahmoud H (2008) Intergration of Municipal Infratrusture Asset Management Processes: Challegnes and Solutions. Journal of Computing in Civil Engineering.

10. Nemati HR, Steiger DM, Lyer LS, Herschel RT (2002) Knowledge warehouse: an architectural integration of knowledge management, decision support, artificial intelligence and data warehousing, pp: 143-161.

11. Provance (2014) IT Asset Management: It's All About Process. IT Asset Management for Microsoft System Center. 